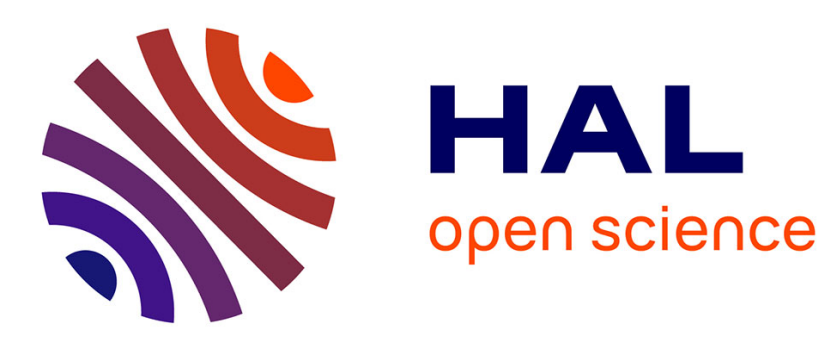

\title{
Coverability in Two Dimensions
}

Guilhem Gamard, Gwenaël Richomme

\section{To cite this version:}

Guilhem Gamard, Gwenaël Richomme. Coverability in Two Dimensions. 9th International Conference on Language and Automata Theory and Applications (LATA), Mar 2015, Nice, France. pp.402-413, 10.1007/978-3-319-15579-1_31. lirmm-01180026

\section{HAL Id: lirmm-01180026 https://hal-lirmm.ccsd.cnrs.fr/lirmm-01180026}

Submitted on 28 Jun 2019

HAL is a multi-disciplinary open access archive for the deposit and dissemination of scientific research documents, whether they are published or not. The documents may come from teaching and research institutions in France or abroad, or from public or private research centers.
L'archive ouverte pluridisciplinaire HAL, est destinée au dépôt et à la diffusion de documents scientifiques de niveau recherche, publiés ou non, émanant des établissements d'enseignement et de recherche français ou étrangers, des laboratoires publics ou privés. 


\title{
Coverability in Two Dimensions
}

\author{
Guilhem Gamard ${ }^{1}$ and Gwenaël Richomme ${ }^{12}$ \\ 1 LIRMM (CNRS, Univ. Montpellier 2) \\ UMR 5506, CC 477, 161 rue Ada, 34095, Montpellier Cedex 5, France \\ \{guilhem.gamard, gwenael.richomme\}@lirmm.fr \\ 2 Univ. Paul-Valéry Montpellier 3 \\ Dpt MIAp, Route de Mende, 34199 Montpellier Cedex 5, France
}

\begin{abstract}
A word is quasiperiodic (or coverable) if it can be covered by occurrences of another finite word, called its quasiperiod. This notion was previously studied in the domains of text algorithms and combinatorics of right infinite words. We extend several results to two dimensions. We also characterize all rectangular words that cover non-periodic two-dimensional infinite words. Then we focus on two-dimensional words with infinitely many quasiperiods. We show that such words have zero entropy. However, contrarily to the one-dimensional case, they may not be uniformly recurrent.
\end{abstract}

Keywords: Combinatorics on Words, Patterns

\section{Introduction}

At the beginning of the 1990's, in the area of text algorithms, Apostolico and Ehrenfeucht introduced the notion of quasiperiodicity [1]. Their definition is as follows: "a string $w$ is quasiperiodic if there is a second string $u \neq w$ such that every position of $w$ falls within some occurrence of $u$ in $w$ ". The word $w$ is also said to be $u$-quasiperiodic, and $u$ is called a quasiperiod (or a cover) of $w$. For instance, the string:

$$
\text { ababaabababaababababaababa }
$$

is $a b a$-quasiperiodic and $a b a b a$-quasiperiodic.

In 2004, Marcus extended this notion to right-infinite words and observed some basic facts about this new class. He opened several questions [10], most of them related to Sturmian words and the subword complexity. First answers were given in [7]. A characterization of right-infinite quasiperiodic Sturmian words was given in [8] and extended to episturmian words in [5]. More details on the complexity function were given in $[11,12]$.

In [11], Marcus and Monteil showed that quasiperiodicity is independent from several other classical notions in combinatorics on words. They also introduced a stronger notion of quasiperiodicity, namely multi-scale quasiperiodicity, with better properties.

Finally, in [4], the authors introduced a two-dimensional version of quasiperiodicity. In particular, they gave a linear-time algorithm computing all square 
quasiperiods of a given square matrix of letters. Our approach is to continue the study of two-dimensional quasiperiodicity by generalizing the results from [11] to infinite two-dimensional words.

- First, we recall definitions of some classical notions from combinatorics on words in a two-dimensional context. Then, to illustrate these notions and quasiperiodicty, we check that independence between these notions and quasiperiodicity is still true in two dimensions (Section 2).

- We determine a necessary and sufficient condition for a word to be a quasiperiod of non-periodic two-dimensional word. Given a quasiperiod $q$, we construct a substitution allowing to forge $q$-quasiperiodic words with various properties, in particular aperiodicity (Section 3).

- We define multi-scale quasiperiodicity in two dimensions. Then we study how multi-scale quasiperiodicity is linked to other classical notions from combinatorics on words. (Section 4).

Warning. Note that in some contexts, most notably in the field of tilings, "quasiperiodic" means "uniformly recurrent". Hence we refer to quasiperiodic words as coverable words; each quasiperiod is a cover (or covering pattern).

\section{Coverability}

Let $\Sigma$ be a finite alphabet. A two-dimensional word (or $\mathbb{Z}^{2}$-word) is a function from $\mathbb{Z}^{2}$ to $\Sigma$. Unless otherwise stated, those functions are assumed to be total. When clarification will be needed, we will note $\operatorname{dom}(\mathbf{w})$ the domain of $\mathbf{w}$, i.e. the set of coordinates where it has defined letters.

A rectangular word is a word $w$ such that $\operatorname{dom}(w)=\{i, \ldots, i+n\} \times\{j, \ldots, j+$ $m\}$, for $i, j \in \mathbb{Z}$ and $n, m \in \mathbb{N}$. In that case, let $\operatorname{width}(w)=n+1$ and height $(w)=$ $m+1$. The set of rectangular words of dimension $n \times m$ is $\Sigma^{n \times m}$. More generally, if $u$ is a rectangular word, then $u^{n \times m}$ denotes the $n \operatorname{width}(u) \times m$ height $(u)$ rectangle which consists only in occurrences of $u$.

Let $\mathcal{C}_{\Sigma, n}$ denote the set of $n$-columns over $\Sigma$, i.e. $1 \times n$-rectangular words over $\Sigma$. Those columns are concatenated horizontally. Likewise, let $\mathcal{L}_{\Sigma, m}$ denote the set of $m$-lines over $\Sigma$, concatenated vertically. We will occasionally view rectangular words as finite one-dimensional words over $\mathcal{C}_{\Sigma, n}$ or $\mathcal{L}_{\Sigma, m}$, considered as alphabets.

In what follows, let $\mathbf{w}$ be a bidimensional word and let $u, v$ be rectangular words.

We say that $u$ is a cover (or a covering pattern) of $\mathbf{w}$ if, for all $(x, y) \in \mathbb{Z}^{2}$, there exists $(i, j) \in \mathbb{N}^{2}$ with $0 \leq i<\operatorname{width}(u)$ and $0 \leq j<\operatorname{height}(u)$ such that $\mathbf{w}[x-i \ldots x-i+\operatorname{width}(u)-1 ; y-j \ldots y-j+\operatorname{height}(u)-1]$ is equal to $u$ up to shift. Intuitively, $u$ is a cover of $\mathbf{w}$ when each position of $\mathbf{w}$ belongs to an occurrence of $u$.

Now we recall some classical notions from combinatorics on words, adapted to the two-dimensional case. Then we will check that coverability is independent 
from these notions. This will generalize the first part of [11] to two-dimensional words.

Let $\mathbf{w}[i \ldots i+n ; j \ldots j+m]$ denote the restriction of $\mathbf{w}$ to the rectangle $\{i \ldots i+n\} \times\{j \ldots j+m\}$, for $i, j \in \mathbb{Z}$ and $n, m \in \mathbb{N}$. If $u=\mathbf{w}[i \ldots i+n ; j \ldots j+m]$ for some $i, j, n$ and $m$, then $u$ is a block of $\mathbf{w}$.

A two-dimensional word $\mathbf{w}$ is uniformly recurrent if, for all $k \in \mathbb{N}$, there exists some $\ell \in \mathbb{N}$ such that all $k \times k$-blocks of $\mathbf{w}$ appear in all $\ell \times \ell$-blocks of $\mathbf{w}$. Intuitively, this means that any block of $\mathbf{w}$ appears infinitely often with bounded gaps.

Moreover, a two-dimensional word $\mathbf{w}$ has a vector of periodicity $\vec{x} \in \mathbb{Z}^{2 *}$ if, for all vectors $\vec{y} \in \mathbb{Z}^{2}$, we have $\mu(\vec{x})=\mu(\vec{x}+\vec{y})$. We say that $\mathbf{w}$ is periodic if it has at least two non-colinear vectors of periodicity. Links between periodicity and others notion defined in this section (most notably the block complexity function, see below) are currently investigated, see e.g. [3].

Let $c_{\mathbf{w}}(n, m)$ be the number of $n \times m$-blocks of $\mathbf{w}$ ( $c_{\mathbf{w}}$ is known as the block complexity function of $\mathbf{w}$ ). Then the the topological entropy of $\mathbf{w}$ is the following quantity:

$$
H(\mathbf{w})=\lim _{n \rightarrow \infty} \frac{\log _{|\Sigma|} c_{\mathbf{w}}(n, n)}{n^{2}}
$$

Intuitively, if $c_{\mathbf{w}}(n, n) \simeq|\Sigma|^{\epsilon n^{2}}$, then $H(\mathbf{w}) \simeq \epsilon$. In other words, when the complexity function of $\mathbf{w}$ is polynomial, $\mathbf{w}$ has zero entropy. This is a classical regularity property on words, often used in the context of dynamical systems.

Let $|u|_{v}$ denote the number of occurrences of $v$ in $u$. The frequency of $u$ in $\mathbf{w}$ is the following quantity:

$$
f_{\mathbf{w}}(u)=\lim _{n \rightarrow \infty} \frac{|\mathbf{w}[-n \cdots+n,-n \cdots+n]|_{u}}{n^{2}}
$$

when it exists. If $f_{\mathbf{w}}(u)$ exists for all blocks $u$ of $\mathbf{w}$, then $u$ is said to have frequencies. This is another common regularity property coming from dynamical systems.

Proposition 1. Coverability is independent from uniform recurrence, subword complexity and existence of frequencies.

Proof. For uniform recurrence, observe that $q=\begin{array}{ccc}q & b & a \\ \begin{array}{l}b \\ b\end{array} & b & b \\ a & b & b\end{array}$ is a cover of the nonuniformly recurrent word displayed on Figure 1 . With the same value of $q$, the $q$-periodic two-dimensional word is uniformly recurrent.

Let $\mathbf{w}$ be a two-dimensional word over $\{a, b\}$ with polynomial (resp. exponential) complexity. Consider the following function:

$$
\begin{aligned}
& \nu(a)=a b a b a a b a \\
& \nu(b)=a b a a b a b a
\end{aligned}
$$

The image $\nu(\mathbf{w})$ has polynomial with the same degree (resp. exponential) complexity and is $a b a$-coverable (viewing $a b a$ as a $3 \times 1$-rectangle). 
Finally, the word $\nu\left(a^{\mathbb{Z}^{2}}\right)$ has frequencies for all its blocks. By contrast, if $\mathbf{w}$ is a word having no frequencies for any block, then $\nu(\mathbf{w})$ has no frequencies either.

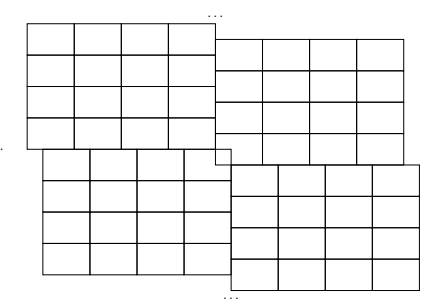

Fig. 1. A coverable, non-uniformly recurrent word

As a conclusion, coverability is a weak notion: it does not bring much information about two-dimensional words it characterizes.

\section{Aperiodic Coverings}

In this section, we determine under which conditions a rectangular word $q$ can be a cover of an aperiodic $\mathbb{Z}^{2}$-word $w$. First, let us consider the question for $\mathbb{N}$-words. Recall that, in this context, a border is a block of $q$ which is both a proper prefix and a suffix of $q$. (A word $u$ is a proper block of $v$ if it is a block of $v$ and $u \neq v)$.

Lemma 2. A finite one-dimensional word $q$ is a cover of an aperiodic coverable $\mathbb{N}$-word if and only if the primitive root of $q$ has a non-empty border.

Sketch of proof. If $q$ is a cover of an aperiodic infinite word, then so is its primitive root. If the primitive root $r$ of $q$ has no non-empty borders, then two occurrences of $r$ never properly overlap. Hence any $r$-covered word must be periodic.

Conversely, if $r$ has a non-empty border and if $q=r^{k}$ for some positive integer $k$, then $r=u v u$. Let $h$ be the morphism defined by $h(a)=(u v u)^{k}$ and $h(b)=(u v u)^{k-1} v u$. The image of any aperiodic word by $h$ is an aperiodic, $r$ coverable word. The proof is omitted by lack of space, but proof of Theorem 5 works quite the same.

The previous result also holds for $\mathbb{Z}$-words. For $\mathbb{N}^{2}$-words, one can prove similarly: a finite rectangular word $q$ is a cover of some aperiodic infinite word if and only if the primitive root of $q$ has a non-empty horizontal border or a non-empty vertical border. In this context, a horizontal (resp. vertical) border is a rectangular word which has the same width (resp. height) as $q$ and which occurs both at the top and the bottom (resp. left and right) of $q$. 
The proof of Lemma 2 used a morphism. Given a word $w$, readers can check that $h(w)$ shares a lot of common properties with $w$. From now on, we focus on the $\mathbb{Z}^{2}$ case. Theorem 5 below generalizes the previous result for $\mathbb{Z}^{2}$-words. However, our deeper goal is to to construct a substitution over $\mathbb{Z}^{2}$ allowing to obtain $q$-coverable $\mathbb{Z}^{2}$-words with various properties.

\subsection{Primitive Roots of Rectangular Words}

We need some simple definitions to state our characterization. Let $q$ and $r$ be rectangular words. By definition, $r$ is a root of $q$ if $q=r^{n \times m}$, for some positive integers $n$ and $m$. If $q$ has no roots except itself, it is said to be primitive.

These notions initially came from combinatorics on one-dimensional words. The following lemma is a classical result about roots in one dimension. It shows that any one-dimensional finite word has a smallest root, called its primitive root.

Lemma 3. (See, e.g., [9], Prop. 1.3.1 and 1.3.2.)

Given any finite one-dimensional words $u$ and $v$, the following statements are equivalent:

1. there exist integers $n, m \leq 0$ with $(n, m) \neq(0,0)$, such that $u^{n}=v^{m}$;

2. there exist a word $t$ and positive integers $k$ and $\ell$ such that $u=t^{k}$ and $v=t^{\ell}$;

3. $u v=v u$.

Let us show that primitive roots are also well-defined on rectangular words.

Lemma 4. Let $q$ be a rectangular word. Suppose that $q$ has two distinct roots $r_{1}$ and $r_{2}$. Then there exists a rectangular word $r_{3}$ such that $r_{3}$ is a root of both $r_{1}$ and $r_{2}$.

Proof. Let $r_{1}^{k}$ (resp. $r_{2}^{k}$ ) denote $k$ occurrences of $r_{1}$ (resp. $r_{2}$ ) concatenated vertically. Since $r_{1}$ and $r_{2}$ are roots of $q$, there exist integers $n$ and $m$ such that both $r_{1}^{n}$ and $r_{2}^{m}$ are roots of $q$, with height $(q)=\operatorname{height}\left(r_{1}^{n}\right)=\operatorname{height}\left(r_{2}^{m}\right)$. Consider $q, r_{1}^{n}$ and $r_{2}^{m}$ as words over $\mathcal{C}_{\Sigma \text {,height }(q)}$; by Lemma 3 , there exists a word $c$ over $\mathcal{C}_{\Sigma \text {,height }(q)}$ such that $c$ is a root of both $r_{1}^{n}$ and $r_{2}^{m}$.

Let $r_{3}$ (resp. $r_{4}$ ) be the horizontal prefix of $r_{1}$ (resp. $r_{2}$ ) of length width $(c)$. Both $r_{3}$ and $r_{4}$ are prefixes of $q$, hence $r_{3}^{n}=r_{4}^{m}$ (the power is still taken for verti-

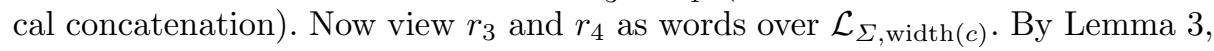
there exists a word $r$ over $\mathcal{L}_{\Sigma \text {,width(c) }}$ which is a common root of $r_{3}$ and $r_{4}$.

As $r_{1}$ (resp. $r_{2}$ ) is obtained by horizontal concatenations of occurrences of $r_{3}$ (resp. $r_{4}$ ), we deduce that $r$ is a root of $r_{1}$ and of $r_{2}$.

The primitive root of a rectangular word $q$ is the root minimal for the "is a root of" relation. By Lemma 4, it is the only root of $q$ (possibly itself) which is primitive.

We need one last definition before stating our first theorem. Let $q$ be a rectangular word. Following [4], a proper block $b$ of $q$ is a diagonal border of $q$ if $b$ occurs in two opposite corners of $q$. Note that it is possible to have either $\operatorname{width}(b)=\operatorname{width}(q)$ (horizontal border) or height $(b)=\operatorname{height}(q)$ (vertical border), but not both. 


\subsection{Patterns Covering Aperiodic Bidimensional Words}

Now we can state the condition under which a rectangular word can be the covering pattern of a non-periodic $\mathbb{Z}^{2}$-word.

Theorem 5. Let $q$ be a finite, rectangular word. Then there exists a q-coverable, non-periodic $\mathbb{Z}^{2}$-word if and only if the primitive root of $q$ has a non-empty diagonal border.

This subsection is entirely dedicated to the proof of Theorem 5 .

Proof of the "only if" part. First, suppose that $\mathbf{w}$ is a $\mathbb{Z}^{2}$-word which is both $q$-coverable and non-periodic. There exists at least two overlapping occurrences of $q$ in $\mathbf{w}$. Moreover, the overlapping part is not a power of the primitive root of $q$ : if all overlappings are powers of some root of $q$, then $\mathbf{w}$ is periodic. Therefore, $q$ must have at least one border which is not a power of its primitive root. Hence its primitive root has a non-empty border.

Proof of the "if" part. Suppose that $q$ 's primitive root has a non-empty diagonal border. Let us build an infinite $\mathbb{Z}^{2}$-word which is $q$-coverable, but not periodic.
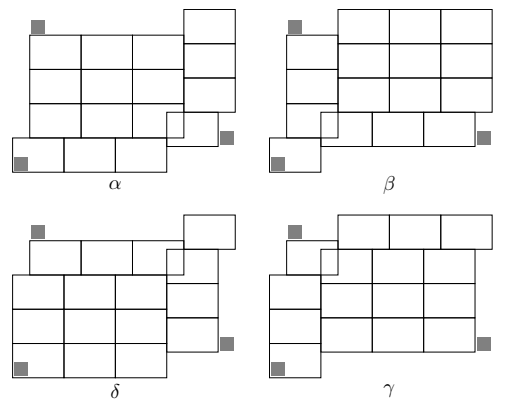

Fig. 2. Four tiles to build a $q$-coverable word. Each rectangle is an occurrence of $q$

Let $r$ be the primitive root of $q$ and $b$ be a non-empty diagonal border of $r$. Consider the four tiles $\alpha, \beta, \delta$ and $\gamma$ displayed on Figure 2. Each rectangle is an occurrence of $q$. The overlapping zones are all occurrences of $b$ and the shifts on tile borders are sized accordingly. If the border $b$ is on the opposite corner, all tiles are built symmetrically.

Let $A=\left\{a_{1}, a_{2}, a_{3}, a_{4}\right\}$ and $\mu$ be the function from $A^{\mathbb{Z}^{2}}$ to $\Sigma^{\mathbb{Z}^{2}}$, defined by $\mu\left(a_{1}\right)=\alpha, \mu\left(a_{2}\right)=\beta, \mu\left(a_{3}\right)=\gamma$ and $\mu\left(a_{4}\right)=\delta$. If its input is regular enough, $\mu$ behaves more or less like a morphism, with the following concatenation rules.

On Figure 2, each tile has three anchors, i.e. letters marked by a small square. Concatenate two tiles horizontally by merging the right-anchor of the first one with the left-anchor of the second one. Concatenate two tiles vertically by merging the bottom-anchor of the first one with the top-anchor of the second one. 
More formally, we have:

$$
\begin{aligned}
\mu\left(a_{i} \cdot u\right) & =\mu\left(a_{i}\right) \cup S_{(4 \operatorname{width}(q) ; \operatorname{height}(b))} \circ \mu(u) \\
\mu\left(\begin{array}{l}
u \\
v
\end{array}\right) & =\mu(v) \cup S_{(\operatorname{width}(b) ; 4 \operatorname{height}(q))} \circ \mu(u)
\end{aligned}
$$

where the operator $\cup$ denotes the superposition of two finite words. Recall that we view two-dimensional words as (possibly partial) functions from $\mathbb{Z}^{2}$ to the alphabet. These functions have domains which may be strictly included in $\mathbb{Z}^{2}$. If $w_{1}$ and $w_{2}$ are two words with disjoints domains, then $\left(w_{1} \cup w_{2}\right)[x, y]=w_{1}[x, y]$ where $w_{1}$ is defined and $w_{2}[x, y]$ where $w_{2}$ is defined. In what follows, we will only consider superpositions where no position $(x, y)$ is defined in both $w_{1}[x, y]$ and $w_{2}[x, y]$.

If $u$ is a rectangular word, the leftmost bottom anchor of $\mu(u[i, j])$ has coordinates:

$$
(i \times 4 \times \operatorname{width}(q)+j \times \operatorname{width}(b) ; j \times 4 \times \operatorname{height}(q)+i \times \operatorname{height}(b))
$$

in $\mu(u)$. Figure 3 gives an example of how $\mu$ works.

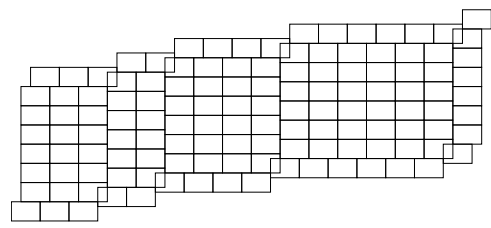

Fig. 3. $\mu\left(\begin{array}{cccc}a_{3} & a_{4} & a_{4} & a_{3} \\ a_{1} & a_{2} & a_{2} & a_{1}\end{array}\right)$, each rectangle is an occurrence of $q$

A word over $A$ is suitable when it satisfies the following conditions:

1. each line is either on alphabet $\left\{a_{1}, a_{2}\right\}$ or on alphabet $\left\{a_{3}, a_{4}\right\}$;

2. each column is either on alphabet $\left\{a_{1}, a_{3}\right\}$ or on alphabet $\left\{a_{2}, a_{4}\right\}$.

First, we check that if $\mathbf{w}$ is suitable, then each letter of $\mu(\mathbf{w})$ belongs to the image of exactly one letter of $\mathbf{w}$. This essentially means that all tiles "fit together" with no overlaps.

By construction, tiles $\alpha$ and $\delta$ fit together vertically, and tiles $\beta$ and $\gamma$ fit as well. Hence $\mu\left(\begin{array}{c}a_{1} \\ a_{3}\end{array}\right)$ and $\mu\left(\begin{array}{c}a_{2} \\ a_{4}\end{array}\right)$ are well-defined. Likewise, tiles $\alpha$ and $\beta$ fit together horizontally, and tiles $\delta$ and $\gamma$ fit as well. Hence $\mu\left(a_{1} a_{2}\right)$ and $\mu\left(a_{3} a_{4}\right)$ and are well-defined. Iterating this argument, we deduce that the image of any suitable word is well-defined.

Moreover, we let readers check that $\mu(\mathbf{w})$ has no "holes". More precisely, if if $\mathbf{w}$ is a suitable rectangular word, $\mu(w)$ satisfies the following weak convexity properties:

- for all $i, j, j_{1}, j_{2} \in \mathbb{N}$ with $j_{1} \leq j \leq j_{2}$, if $\left(i, j_{1}\right)$ and $\left(i, j_{2}\right)$ are in $\operatorname{dom}(\mu(\mathbf{w}))$, then $(i, j)$ is in $\operatorname{dom}(\mu(\mathbf{w}))$ as well; 
- for all $i, j, i_{1}, i_{2} \in \mathbb{N}$ with $i_{1} \leq i \leq i_{2}$, if $\left(i_{1}, j\right)$ and $\left(i_{2}, j\right)$ are in $\operatorname{dom}(\mu(\mathbf{w}))$, then $(i, j)$ is in $\operatorname{dom}(\mu(\mathbf{w}))$ as well.

As a consequence, the definition of $\mu$ can be extended to suitable $\mathbb{Z}^{2}$-words. If $\mathbf{w}$ is a suitable $\mathbb{Z}^{2}$-word, then $\mu(\mathbf{w})$ is a well-defined $\mathbb{Z}^{2}$-word as well.

We will now prove that $\mu(\mathbf{w})$ is aperiodic for any aperiodic bidimensional word w. First, we need a technical lemma about our tiles.

Lemma 6. Let $x$ and $y$ be different tiles from $\{\alpha, \beta, \gamma, \delta\}$. Then an occurrence of $x$ and an occurrence of $y$ cannot overlap when their anchor points coincide.

This essentially means that situations from Figure 4 cannot occur.
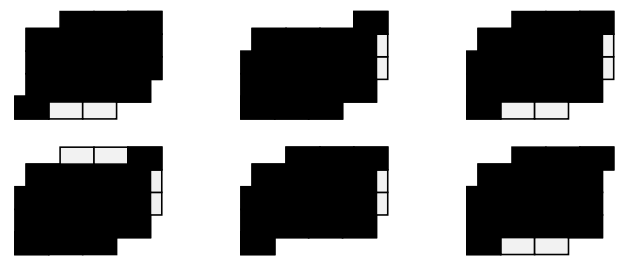

Fig. 4. All other possible overlappings

Proof. There are six possibilities for the set $\{x, y\}$. All proofs are quite similar, so we only provide a proof when $x=\alpha$ and $y=\beta$ (illustrated by the top lefthand case of Figure 4). In what follows, $q$ refers to the rectangular word used for the construction of the tiles, $r$ to its primitive root and $b$ to a diagonal border of $r$.

There are three occurrences of $q$, named $q_{1}, q_{2}$ and $q_{3}$, such that $q_{1}$ is covered by $q_{2}$ and $q_{3}$ and all three are horizontally aligned. (See for instance the top second column of $q$ 's in the figure). View $q_{1}, q_{2}$ and $q_{3}$ as one-dimensional words over the alphabet $\mathcal{L}_{\Sigma, \operatorname{width}(q)}$. There exist words $x$ and $x^{\prime}$ over $\mathcal{L}_{\Sigma, \operatorname{width}(q)}$ such that $q_{1}=x x^{\prime}$ and $q_{2}=q_{3}=x^{\prime} x$ (where words are concatenated from bottom to top).

By Lemma $3, x$ and $x^{\prime}$ (and $q$ ) are powers of a same word $s$ over $\mathcal{L}_{\Sigma \text {,width }(q)}$. Notice that height $\left(x^{\prime}\right)=\operatorname{height}(b)$ and height $(x)=\operatorname{height}(q)-$ height $(b)$. It follows that height $(s)$ divides height $(x)$ and height $(q)-\operatorname{height}(b)$.

Observe that $s$ is a vertical prefix of both $q$ and $x$. Thence one can find three occurrences of $s$, named $s_{1}, s_{2}$ and $s_{3}$, such that $s_{1}$ is covered by $s_{2}$ and $s_{3}$ and all three are vertically aligned. (See for instance the second line of $q$ 's in the figure).

Now view $s$ as a one-dimensional word on the alphabet $\mathcal{C}_{\Sigma \text {,height }(s)}$. There exist words $y, y^{\prime}$ such that $s_{1}=y y^{\prime}, s_{2}=s_{3}=y^{\prime} y$ and $\operatorname{width}\left(y^{\prime}\right)=\operatorname{width}(b)$. By Lemma 3, we deduce that there exists a word $t$ over $\mathcal{C}_{\Sigma \text {,height }(s)}$ such that $y$ and $y^{\prime}$ (and $s$ ) are powers of $t$. 
Let $k \leq 1$ be the integer such that $q=s^{k}$ (for vertical concatenation) and let $\ell \geq 1$ be the integer such that $s=t^{\ell}$ (for horizontal concatenation). We have that $q=t^{\ell \times k}$. Therefore $t$ is a root of $q \operatorname{such}$ that $\operatorname{width}(t) \leq w i d t h\left(y^{\prime}\right)=w i d t h(b)$ and height $(t)=\operatorname{height}(s) \leq \operatorname{height}(b) . \operatorname{Thus} \operatorname{width}(s) \times \operatorname{height}(s) \leq \operatorname{width}(b) \times$ height $(b)$ which is a contradiction with the definition of $b$. Indeed, recall that $b$ is a border (hence a proper block) of the primitive root of $q$, which is smallest (in number of letters) roots of $q$.

In the proof of next lemma, Lemma 6 helps to establish a correspondence between the letters of the $\mathbb{Z}^{2}$-word $\mu(\mathbf{w})$ and the "tiling" consisting of occurrences of $\alpha, \beta, \delta$ and $\gamma$. We need this correspondence to prove that some $\mu(\mathbf{w})$ can always be made aperiodic.

Lemma 7. Let $q$ be a rectangular word, $r$ its primitive root and $b$ one non-empty diagonal border of $r$. Let $\mathbf{w}$ be an aperiodic, suitable $\mathbb{Z}^{2}$-word. Then $\mu(\mathbf{w})$ is an aperiodic, q-coverable $\mathbb{Z}^{2}$-word.

Proof. By construction, $\mu(\mathbf{w})$ is $q$-coverable for all $\mathbf{w}$. Suppose that $\mu(\mathbf{w})$ has a non-null vector of periodicity $\vec{p} \in \mathbb{Z}^{2}$. Let us prove that, under this assumption, $\mathbf{w}$ is periodic.

Let $a \in \mathbb{Z}^{2}$ be the coordinates of the anchor point of some tile in $\mu(\mathbf{w})$. For any $i \in \mathbb{Z}$, let $t_{i}=a+i \times \vec{p}$. Since tiles have at most $16 \times \operatorname{width}(q) \times \operatorname{height}(q)$ letters, by pigeonhole principle, there are two pairs of coordinates $t_{i}$ and $t_{j}$ which have the same offset to the anchor points of their respective tiles (i.e. the tiles covering their respective positions). Hence the difference between these anchor points is a multiple of the vector of periodicity $\vec{p}$.

Let $T_{i}$ (resp. $T_{j}$ ) be the tile covering position $t_{i}\left(\right.$ resp. $\left.t_{j}\right)$. Since $T_{i}$ is the $(j-$ i) $\times \vec{p}$-translation of $T_{j}$, they are both occurrences of a same tile. Moreover, the right-neighbours of $T_{i}$ and $T_{j}$ are both occurrences of a same tile, otherwise we would have a configuration forbidden by Lemma 6 . Likewise, the top-neighbour, bottom-neighbour and left-neighbour of $T_{i}$ and $T_{j}$ are also equal. By iterating this argument over the neighbours' neighbours, and so on, we conclude that the tiling itself is periodic. Hence, $\mathbf{w}$ is periodic.

This ends the proof of Theorem 5. From any rectangular word $q$ with at least one non-empty diagonal border in its primitive root, we can build $\mu(\mathbf{w})$ for any aperiodic, suitable $\mathbb{Z}^{2}$-word $\mathbf{w}$.

\subsection{Lifting other Properties to Coverable Words}

Notice how we "lifted" aperiodicity from an arbitrary $\mathbb{Z}^{2}$-word to a $q$-coverable word. This technique can be used to lift other properties, such as existence of frequencies, uniform recurrence, block complexity or topological entropy. The proof is as in Proposition 1, using $\mu$ instead of $\nu$.

Hence, for any rectangular word $q$, there exist $q$-coverable $\mathbb{Z}^{2}$-words with or without uniform recurrence, with or without frequencies, and with any complexity function. Any rectangle which is the cover of a $\mathbb{Z}^{2}$-word is also the cover of 
$\mathbb{Z}^{2}$-words with any properties. In other terms, the contents of a covering pattern do not bring any information about the covered word.

As a conclusion of this section, remark that our substitution preserves various kind of non-periodic properties. In Lemma 3.5, we could have assumed that there exists no-sequence $\left(x_{i}, y_{i}\right)_{i \in \mathbb{N}}$ of coordinates in $w$ with $\left(x_{i+1}-x_{i}, y_{i+1}-y_{i}\right)$ constant. We would have obtained exactly the same property for $\mu(\mathbf{w})$.

\section{Multi-Scale Coverability in Two Dimensions}

In [11], Monteil and Marcus called multi-scale coverable any $\mathbb{N}$-word having infinitely many covers. We want to exclude cases where coverability is obtained on groups of lines (or columns) stacked all over $\mathbb{Z}^{2}$. Hence our generalization is more specific. A $\mathbb{Z}^{2}$-word (or a $\mathbb{N}^{2}$-word) is called multi-scale coverable if, for each $n \in \mathbb{N}$, it has a $k \times \ell$ cover with both $k \geq n$ and $\ell \geq n$.

In [11], Monteil and Marcus prove that multi-scale coverable right-infinite words have zero entropy and are uniformly recurrent. We study these results for $\mathbb{Z}^{2}$-words.

\subsection{Topological Entropy}

Let $\mathbf{w}$ be a $\mathbb{Z}^{2}$-word. Recall that $c_{\mathbf{w}}(n, m)$ is the number of rectangles of size $n \times m$ which occur in $\mathbf{w}$ and that the topological entropy of $\mathbf{w}$ is the following quantity:

$$
H(w)=\lim _{n \rightarrow+\infty} \frac{\log _{|\Sigma|} c_{w}(n, n)}{n^{2}}
$$

This sequence converges since $\frac{\log _{|\Sigma|} c_{w}(n, n)}{n}$ is sub-additive (thanks to the Fekete's Subadditive Lemma, see e.g. [13]).

Proposition 8. Any multi-scale coverable, $\mathbb{Z}^{2}$-word $\mathbf{w}$ has zero entropy.

Proof. Consider a covering pattern $q$ of $\mathbf{w}$ with size $n \times m$. Suppose without loss of generality that $n \leq m$. Let $s$ be a $m \times m$-square of $\mathbf{w}$. The square $s$ is covered with occurrences of $q$ (which may spill out of $s$ ). The relative position of $s$ and of occurrences of $q$ completely defines $s$.

We need at most $4 m$ occurrences of $q$ to define a covering of $s$. Each occurrence of $q$ must have at least one of its corners in $s$. If some occurrence of $q$ has its bottom right-hand corner in $s$, then no other occurrence of $q$ may have their bottom right-hand corners on the same line of $s$. Otherwise, one of these occurrences would supersede the other one, which would be "useless" in the covering.

Proceed the same way for the other corners and deduce that at most $4 \mathrm{~m}$ occurrences of $q$ (4 per line) uniquely define $s$. Each of these occurrences is uniquely determined by its position of its corner on a line of $s$. There are at most $m$ possibilities for each. Therefore, there are at most $m^{4 m} q$-coverings which define all possible squares $s$. 
This bound on $c_{\mathbf{w}}(m, m)$ allows us to compute the entropy of $\mathbf{w}$. Observe that:

$$
\lim _{m \rightarrow \infty} \frac{\log m^{4 m}}{m^{2}}=\frac{4 m \log m}{m^{2}} \rightarrow 0
$$

Since there are infinitely many covering patterns of $\mathbf{w}$ with growing sizes, there are infinitely many integers $m$ such that $c_{\mathbf{w}}(m, m) \leq m^{4 m}$. Hence equation (2) shows that then topological entropy of $\mathbf{w}$ converges to zero.

Note that since the Kolmogorov complexity is bound by the entropy (see [2]), this result also shows that the Kolmogorov complexity of multi-scale coverable words is zero as well.

\subsection{Uniform Recurrence}

Recall that a $\mathbb{Z}^{2}$-word $\mathbf{w}$ is uniformly recurrent when all its blocks occur infinitely often with bounded gaps. In $\mathbb{N}$-words, multi-scale coverability implies uniform recurrence. Quite surprisingly, this is not true for infinite two-dimensional words.

Consider $q=\begin{array}{lll}b & b & a \\ b & b & b \\ a & b & b\end{array}$ and the word displayed on Figure 1. The central block

$$
\begin{aligned}
& b b b b a \\
& b b b b b \\
& b b a b b \\
& b b b b b \\
& a b b b b
\end{aligned}
$$

occurs only once, hence this word is not uniformly recurrent.

Actually, the problem does not lie in the dimension two, but in the absence of origin. The statement "multi-scale coverability implies uniform recurrence" is true for $\mathbb{N}$-words (see [11]) and $\mathbb{N}^{2}$-words, and false for $\mathbb{Z}$-words and $\mathbb{Z}^{2}$-words.

Here is an example of a $\mathbb{Z}$-word which is multi-scale coverable, but not uniformly recurrent:

$$
{ }^{\omega}(a b) a(a b)^{\omega}=\ldots \text { babababa a babababa } \ldots
$$

Any word matching the $a b a(b a)^{*}$ regular expression is a covering pattern of this word. However, the pattern $a a$ only occur once, hence it is not uniformly recurrent.

Proposition 9. Any multi-scale, $\mathbb{N}^{2}$-word $\mathbf{w}$ is uniformly recurrent.

Proof. This is an adaptation of the proof from [11]. Consider a rectangle $r$ occurring in $\mathbf{w}$. Since $\mathbf{w}$ has arbitrarily large covering patterns and all these patterns occur at the origin, one of these patterns contains $r$ entirely. Hence $r$ occurs whenever the covering patterns occurs, and the latter occurs infinitely many times with bounded gaps.

As a conclusion, uniform recurrence from multi-scale coverability does not generalize to $\mathbb{Z}^{2}$-words. However, the situation as a whole generalizes to two dimensions: the implication is true on words "with origins" $\left(\mathbb{N}, \mathbb{N}^{2}\right)$, and false on words "without" $\left(\mathbb{Z}, \mathbb{Z}^{2}\right)$. 


\section{Conclusion and Future Work}

As a conclusion, let us point out several questions on which we are currently working.

In [11], it is shown that all multi-scale coverable words have uniform frequencies. Although the result seems still true for two-dimensional words, the proof appears to be not directly generalizable.

Moreover, we have the feeling that non-uniformly recurrent coverable $\mathbb{Z}^{2}$ words are pathological cases. We suspect that they are all similar to the one displayed on Figure 1. We are currently working on a full characterization of those words.

Finally, one-dimensional coverable words may be decomposed to a normal form (see [6]). This allows to view one-dimensional coverable coverable words as images of arbitrary words by some morphisms (which depend on the cover). However, there does not seem to exist such normal form for coverable two-dimensional words. Is any $q$-coverable word an image by some kind of substitution?

\section{References}

1. Apostolico, A., Ehrenfeucht, A.: Efficient detection of quasiperiodicities in strings. Theor. Comput. Sci. 119(2), 247-265 (1993)

2. Brudno, A.A.: Entropy and complexity of the trajectories of a dynamical system. Tr. Mosk. Mat. O.-va 44, 124-149 (1982)

3. Cassaigne, J.: Subword complexity and periodicity in two or more dimensions. In: Rozenberg, G., Thomas, W. (eds.) Developments in Language Theory, Foundations, Applications, and Perspectives, Aachen, Germany, 6-9 July 1999. pp. 14-21. World Scientific (1999)

4. Crochemore, M., Iliopoulos, C.S., Korda, M.: Two-dimensional prefix string matching and covering on square matrices. Algorithmica 20(4), 353-373 (1998)

5. Glen, A., Levé, F., Richomme, G.: Quasiperiodic and lyndon episturmian words. Theor. Comput. Sci. 409(3), 578-600 (2008)

6. Iliopoulos, C.S., Mouchard, L.: Quasiperiodicity: From detection to normal forms. Journal of Automata, Languages and Combinatorics 4(3), 213-228 (1999)

7. Levé, F., Richomme, G.: Quasiperiodic infinite words: Some answers (column: Formal language theory). Bulletin of the EATCS 84, 128-138 (2004)

8. Levé, F., Richomme, G.: Quasiperiodic sturmian words and morphisms. Theor. Comput. Sci. 372(1), 15-25 (2007)

9. Lothaire, M.: Combinatorics on Words. Cambridge Mathematical Library, Cambridge University Press (1997)

10. Marcus, S.: Quasiperiodic infinite words (columns: Formal language theory). Bulletin of the EATCS 82, 170-174 (2004)

11. Monteil, T., Marcus, S.: Quasiperiodic infinite words: multi-scale case and dynamical properties, http://arxiv.org/abs/math/0603354

12. Polley, R., Staiger, L.: The maximal subword complexity of quasiperiodic infinite words. In: McQuillan, I., Pighizzini, G. (eds.) Proceedings Twelfth Annual Workshop on Descriptional Complexity of Formal Systems, DCFS 2010, Saskatoon, Canada, 8-10th August 2010. EPTCS, vol. 31, pp. 169-176 (2010)

13. Schechter, E.: Handbook of Analysis and Its Foundations. Elsevier Science (1996) 